\title{
Tooth Agenesis; Aetiological Factors
}

\section{Abdulgani Azzaldeen, Nezar Watted, Abdulgani Mai, Péter Borbély, Muhamad Abu-Hussein*}

\begin{abstract}
Tooth agenesis is the most prevalent craniofacial congenital anomaly in humans. The term refers to an isolated disorder in the absence of non-dental phenotypes but is also used to describe the manifestation of missing teeth in syndromes. The affected individuals suffer from compromised masticatory functions and have decreased quality of life. Discerning the genetic etiology of tooth agenesis not only improves our understanding of normal tooth development but also provides a fundamental basis for developing potential therapeutic strategies for this anomaly. To date, MSX1, Pax9, Axin2, Eda, And Wnt10a have been established as candidate genes associated with non-syndromic tooth agenesis.This article reviews the recently discovered genes involved in dental agenesis, and provides an update on the aetiological factors underlying this common malformation.
\end{abstract}

Keywords: etiology, hypodontia, prevalence, risk factors

\section{Introduction}

Hypodontia (dental agenesis) is the most common developmental anomaly in humans, constituting a clinically challenging problem. Hypodontia is often used as a collective term for congenitally missing teeth, although specifically, it describes the absence of one to six teeth, excluding third molars. Oligodontia (multiple aplasia) refers to the congenital absence of six or more teeth, excluding third molars. Anodontia represents a complete failure of one or both dentitions to develop [1] Fig. 1,2

Tooth development can be affected by either environmental or genetic factors. Children with malignant diseases at tooth-developing ages are at high risk of tooth agenesis from treatment. Irradiation therapy has more severe effects on tooth development than chemotherapy . [2] Many genes have been found to affect tooth development through gene expression and experimental studies in mice. In theory, any of these genes may cause tooth agenesis . Family studies show that tooth agenesis, in an isolated form, can be caused by mutations in the MSX1, PAX9, AXIN2, EDA, WNTA10 and SMOC2 genes, with autosomal and X link traits of inheritance. As the phenotypes and genotypes of tooth agenesis are diverse, it is the aim of the study to determine the type of inheritance and phenotype, genotype of familial tooth agenesis among consanguineous families.[2,3]

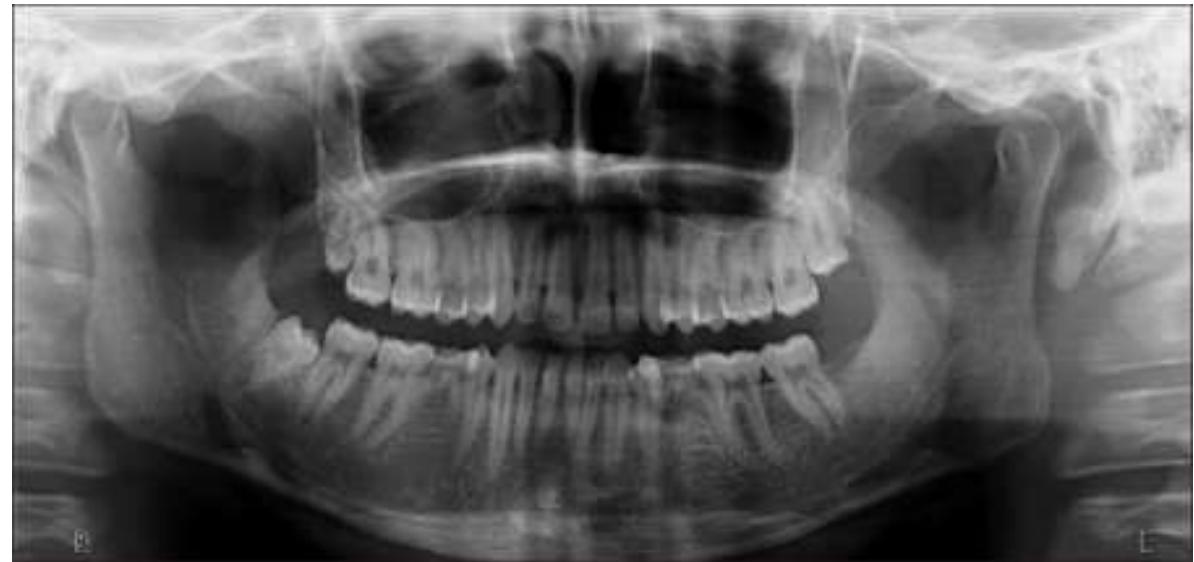

Figure 1: OPG shows the absence of tooth buds 35,45 and also note the complete absence of tooth bud of 38

Tooth agenesis in deciduous dentition is less common than in permanent dentition, with a prevalence of less than $1 \%$ in the general population with the most commonly affected tooth type being the incisor. [4]The upper lateral deciduous incisors are the most commonly missing, accounted for $50 \%$ of the missing deciduous teeth, followed by the lower incisor; both together are accounted for $90 \%$ of all affected deciduous teeth The prevalence of deciduous tooth agenesis seems to be more common in Japan as it has been reported to have a high prevalence of $2.4 \%$, with the most affected tooth being the lower lateral incisor.[4,5]

The severe phenotype of tooth agenesis is rare with a prevalence of only $0.16 \%$ in a Danish study . [6] The prevalence of permanent selective tooth agenesis ranges from $1.6 \%$ to $9.6 \%$ in the general population 
excluding third molars, and it varies with tooth type . [4]Including the third molars, the prevalence is higher, up to $25 \%$ of all reported cases [7]However, these studies on the prevalence of tooth agenesis were carried out on varied populations and ethnic origins. The prevalence in Arabs population in Israel with a 2,6\% incidence which is on the higher limit of the global range $(1.6-9.6 \%)$.[8,9] Severe tooth agenesis is a rare congenital defect. In European populations, it was seen at a level of around $0.25 \%$. [10] The prevalence was found to be $0.08 \%$ in a Dutch study , [11] and $0.16 \%$ in a Danish study . [6]In Japan, the prevalence was found to be slightly higher, about $1.4 \%$.[12]

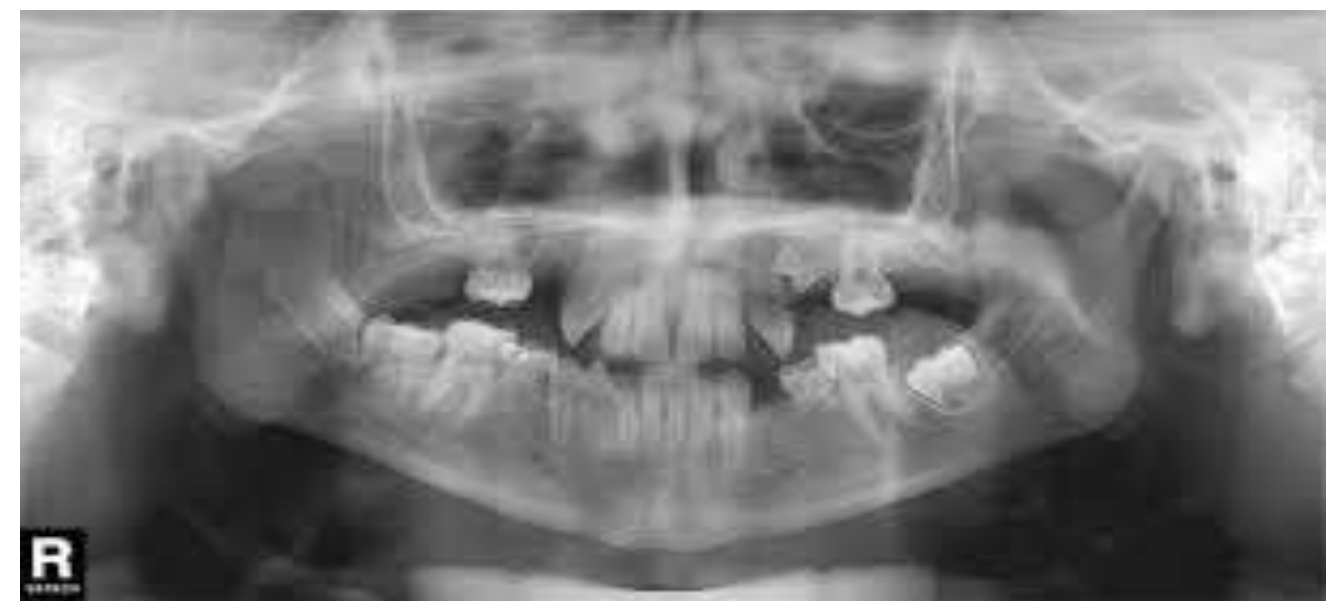

Fig. 2. Panoramic radiograph of the patient. Note the absense of eight teeth and the small dimension in maxilla.

Regarding gender, this anomaly appears more often to women than to men . Some other studies report not any significant statistical difference between genders. .[13,14]Hypodontia refers to the condition where there is an absence of fewer than six teeth. The term Oligodontia is usually used to describe a larger number of missing teeth (six or more). Anodontia is the complete absence of teeth. Many other researchers have used similar methods of classifying the congenital absence of teeth, [15] In general, they identify three categories of hypodontia, excluding third molars, as follows: Mild with 1 or 2 missing teeth. Moderate with 3 - 5 missing teeth. Severe with 6 or more missing teeth. Hypodontia is also classified as either isolated hypodontia or syndromic hypodontia. Isolated hypodontia refers to those cases without syndromes . [4] Thus, hypodontia can occur either as part of a syndrome or as a non-syndromic, familial form; in the latter it occurs as an isolated trait, affects variable numbers of teeth and appears either sporadically or as an inherited condition within a family pedigree. [16] The congenital absence of teeth can seriously affect a young person, both physically and emotionally particularly when the missing tooth is located in the anterior region of the mouth [1]. Early detection of hypodontia may allow a more favorable prognosis and minimal functional, esthetical and psychological complications .[17] Fig. 3

Tooth agenesis is an interesting complex disorder, and in recent decades several theories have been suggested to explain it and verify its aetiology. Currently the aetiology of tooth agenesis is related generally to the role of genetic and environmental factors .[3]

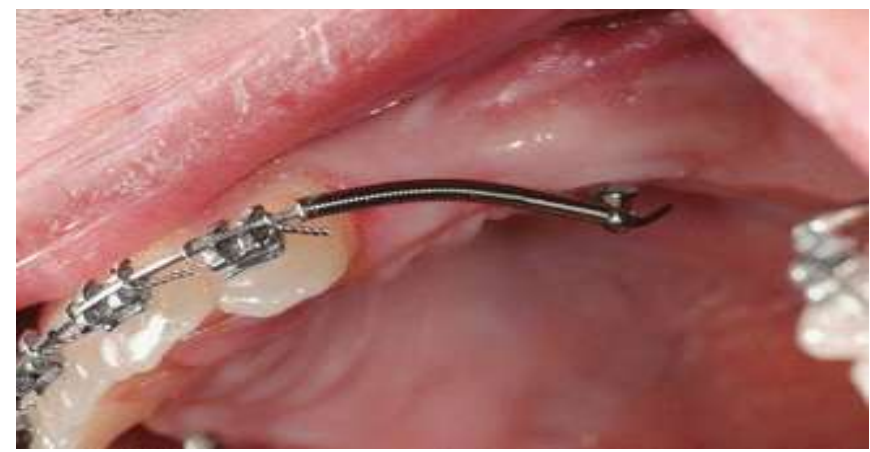

Fig. 3.In patients missing multiple teeth, temporary anchoring devices (TADs) which are really temporary nonintegrating implants are often used during orthodontic therapy

\section{Genetic factors}


Tooth agenesis is found more commonly among individuals related to hypodontia patients than in the population in general56 identifying it as a genetic disease. An exhaustive study on a Swedish family with 685 family members, including 171 probands affected with hypodontia, showed that hypodontia involving permanent teeth is primarily determined by genetic factor(s) . [18] The frequency of hypodontia among races varies.[19] and greater concordance of hypodontia is apparent in identical twins than nonidentical with no environmental etiology apparent in afflicted individuals. [20,21]Familial hypodontia is reported to exhibit mainly autosomal dominant inheritance with incomplete penetrance and variable expressivity. [22] However, an autosomal recessive mode of inheritance for hypodontia has been reported in a Pakistani family which mapped to chromosome 16q12.153 and in another report on Finnish patients that are afflicted with a specific type of hypodontia , where patients notably lacked both deciduous and permanent incisors . [23] It has also been suggested that it can follow sex-linked or polygenic inheritance patterns54- [24]. Recently, direct evidence was gathered for the genetic basis of tooth agenesis thanks to the mapping of human disease genes using linkage analysis followed by mutation analysis of positional candidate genes present in the candidate interval. Using this gene mapping strategy, autosomal dominant hypodontia has been localized to at least three chromosomal loci to date; MSX1 , PAX9 and an unknown locus on chromosome . [5] Five mutations have thus far been identified within MSX1 and ten within PAX9 with both genes also having been found to be deleted in separate studies of familial hypodontia [25] Table 1

The several genes that are pivotal in In this respect, several genes that are pivotal in initiating the development of teeth have been subjected to intense study in the past decade. Mutations in a number of genes were found to interrupt tooth development in mice.[26] However, to date there are only three genes associated with the nonsyndromic form of human tooth agenesis: AXIN2, Msx1, and Pax9. Among them, Msx1 and Pax9 was more intensively studied. Recently, the general structure of the Pax paired domain was described and the phylogenetics and relation between the several members of the Pax family were established. In addition, both gene expression and molecular pathogenesis of Msx1 and Pax9 have been relatively well characterized, making it a special candidate to explain at least part of primate tooth variation.[27]

\begin{tabular}{ll}
\hline Defective gene & Type of tooth agenesis \\
\hline Dix-1 and Dlx-2 & Upper molars \\
$\begin{array}{l}\text { Activin bA, activin } \\
\text { receptors IIA and IIB, } \\
\text { Smad2 }\end{array}$ & Incisors and lower molars \\
Fgf8 & All but lower incisors \\
Msx1 & All \\
Pax9 & All \\
\hline \multicolumn{2}{c}{ Table 1: Genes and the type of tooth agenesis seen in mice }
\end{tabular}

Table 1: Genes and the type of tooth agenesis seen in mice

Among the transcriptional regulatory genes required for tooth formation, the Msx1 homeobox gene is highly expressed in the dental mesenchyme and is essential for tooth development, since targeted gene disruption results in arrested tooth formation at an early stage in Msx1. [27.28]In addition to its expression in the tooth primordia, Msx1 expression is prominent in regions of epithelialmesenchymal interactions in several other embryonic structures, including other craniofacial structures and the limb. These findings have led to the hypothesis that Msx1is an important component in the signalling events that occur between epithelial and mesenchymal tissues.[28]

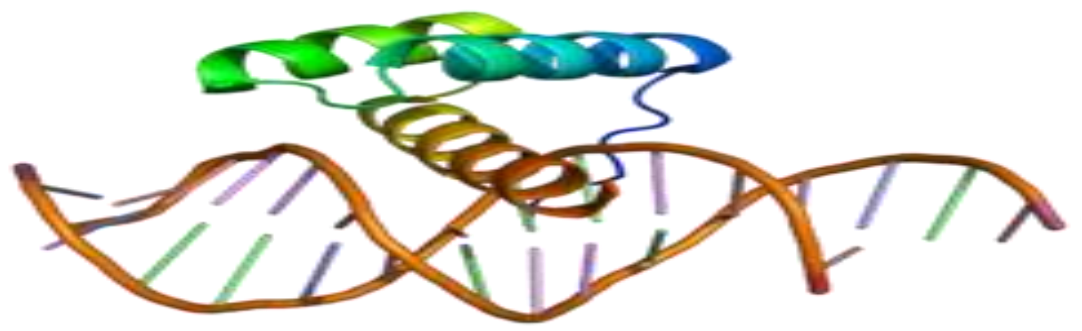

Fig.4. MSX1

The reduced condensation which is also seen in the Pax9 hypomorphic mutans, perhaps indicating a decreased amount of committed dental mesenchymal cells, may be related to tooth agenesis. [29] As Msx1 is known to be important for the commitment of neural crest, an early defect in the migration of neural crest cells 
could also be responsible for the tooth agenesis, if it caused a reduction in the amount of competent ectomesenchymal cells.[30] Fig.4.

The originally expressed throughout the oral epithelium and epidermis, but becomes limited to the placodes at an early stage. When Eda was over expressed in the epithelium, the hair and tooth placodes become larger, probably due to an increased amount of the cells destined to become placode cells. [31] Thus Eda signalling probably acts rather as a modulator of ectodermal placodeformation than as an initiator. [32]Eda signalling may be important as a mediator of effects of Shh and BMPs. Mutation in the Eda and Edar genes in human cause X-linked and autosomal anhidrotic ectodermal dysplasia characterized by failure of sweat development, tooth agenesis and size reduction of teeth.[31,32,33]

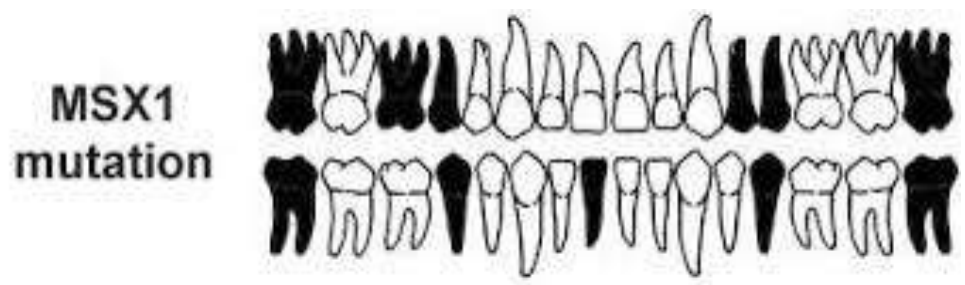

Table 2. Msx1 Mutation

The number and type of teeth are strictly controlled during odontogenesis. Msx1 and Pax9 form a signalling cascade during tooth development. Mutations in Msx1 and Pax9 genes are dominant for tooth agenesis in humans. [34]The gene Pax9 was found to be localized in chromosome 14 (14q12-q13). The disruption of DNA-binding ability of Pax9 that causes hypodontia. Nonsense mutation in exon 1 of Msx 1 in chromosome 4 was found to be heterozygous in all affected family members. Nieminen have identified there was gene deletions in Msx1 and Pax9, missense mutation R196P of Msx1 and missense L21P of Mutation.[35,36]

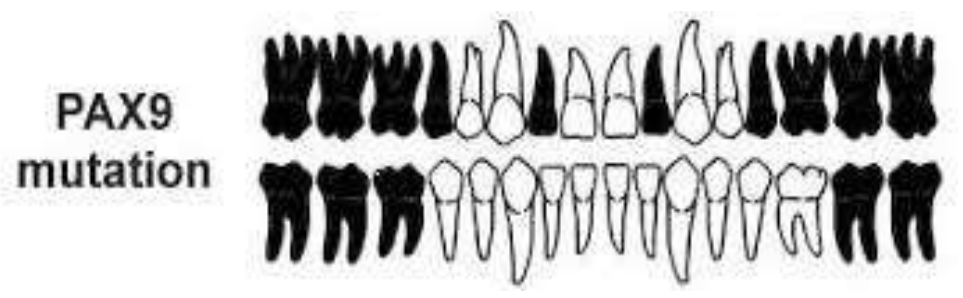

Table 3. Pax9 Mutation

The role of Msx1 and Pax9 is to facilitate the bud to cap stage transition. Mesenchymal Msx 1 expression is initially activated by the epithelial BMP4 signal. Loss of function defects in Msx1 and Pax9 in humans cause partial failure of tooth development, tooth agenesis.[38,39] Defects in Msx1 associate especially with agenesis of second premolars and third molars, whereas the defects in Pax9 affect particularly the permanent molars.[40] The size of the permanent teeth may also be reduced. In one of the families with a defect in Msx1, some patients also presented with nail dysplasia and in another family with oral clefts. Several other sequence changes in Msx1 have also been described in connection with oral clefting. [41,42] In addition, a micro satellite allele in the intron of Msx1 has been associated with both tooth agenesis and oral clefting, and two promoter region SNP alleles of Pax9 with tooth agenesis.[37.43,44] Table 2

Msx1 and Pax9, tooth agenesis has been related to critical function of the mouse homologues of these genes in the formation of the enamel knot and the subsequent transition from bud to cap stages. [45]The Msx1 haploinsufficiency, however, appear to affect only secondary teeth and permanent molars, and it is not obvious how a weakened enamel knot function, which presumably follows from a reduced amount of functional Msx1 protein, is linked to impaired secondary tooth development. [46] It is possible that the late developing teeth are more sensitive to impaired enamel knot function. The development of these teeth normally is a long lasting process and happens surrounded by the alveolar bone. It can also be speculated that enamel knots may regulate the program leading to the secondary tooth formation.[47.48]

Tooth agenesis is a consequence of a qualitatively or quantitatively impaired function of genetic networks, which regulate tooth development. Reduced amount of functional Msx1 or Pax9 protein in the tooth forming cells is able to cause severe and selective tooth agenesis. [49] Another conclusion, based on the analysis of the phenotypes associated with the known defects in these genes, is that the phenotypes associated with the 
defects in Msx1 and those associated with the defects in Pax9 are different. Despite the similarities, there are clearcut differences in the frequency of agenesis of specific teeth.[50] Table 3

\section{Environmental factors}

The sensitivity of tooth development to environmental disturbances has been shown in many studies. Many environmental factors might affect and arrest tooth development such as traumatic dental injuries, cancer therapy and dioxin. The direct relation between tooth development aberration and childhood radiotherapy, chemotherapy and stem cell transplantation has been confirmed. [51]The dental defects may include tooth agenesis, microdontia, developmental delays, root anomalies and calcification defects . The severity of defects and number of teeth involved in this environmental insult are influenced by the age of the child when he/she received the treatment.[52]

The effect of dioxin on tooth development has been reported as an increase in prevalence of hypomineralisation of teeth in Kotka and Anjalankoski in Finland and as an increase in prevalence of tooth agenesis and hypomineralised enamel among people exposed to it in Seveso, Italy . [53]This has been verified in the laboratory in experimental animals . [54]Bailit believed that prenatal environmental factors such as nutrition and diseases have more influence than the postnatal factors on dental development .[55] No specific aetiological relationship has been found between tooth and systemic diseases or endocrine disturbances . [56]This thesis focuses on genetic factors as an explanation of tooth agenesis in the cases reported.

\section{Polygenic factors}

The multifactorial or polygenic model has been proposed, as the tooth agenesis phenotype is very variable, which means that it is not entirely due to a single gene defect.[57] This observation has been evidenced in monozygotic twins and triplets . There is no dominance or recessivity in the polygenic model. [58]Many assumptions have been suggested to simplify this model. For example, each contributing gene has equal effects that are too small to pass a stringent genome-wide significance level, but their additive effects result in phenotype expression. As these genes interact as an incomplete dominance model, there is no linkage involved and large sample sizes are required, thus many collaborations have been established to prove this concept. Genome-wide association studies (GWAS) have provided a great attempt to discover hundreds of genetic variants that are involved in complex diseases and traits such as rheumatoid arthritis and cardiovascular disease . [58]Two statistical analyses have been used to assess the involvement of common SNPs that are below the genome-wide significance level: polygenic analysis and mixed linear modelling, these methods test many SNPs in aggregate for collective effect on phenotype .[59]

\section{Theories About Tooth Agenesis}

Tooth agenesis as a dental defect has been an active area of research; in the last century scientists tried to apply evolutionary and anatomic models to the developmental failure of teeth, for example, odontogenic polarity, Butler's field theory, the anatomical model and Sofaer's model of compensatory tooth size interactions .[4] The theories are described in this thesis in chronological order to show the attempt that has been made during these years to investigate this condition.

\section{Butler's Theory}

Butler's theory (1939) attempts to explain why certain teeth fail to form more than others. According to this hypothesis, mammalian dentition can be divided into 3 morphologic fields corresponding to incisors, canines, and premolars/molars. Within each field, one "key" tooth is presumed to be stable; flanking teeth within the field become progressively less stable. Considering each quadrant separately, the key tooth in the molar/premolar field would be the first molar. This schema positions the second and third molars at the distal end of the field, and the first and second premolars on its mesial end. Based on Butler's theory, the third molar and the first premolar would be predicted to be most variable in size and shape. Clinical epidemiology supports this view for the third molar, but not for the first premolar. However, the earliest mammals had 4 premolars, whereas some higher primates, including man, have lost try sense could be considered unstable. $[1,4,50,55]$

\section{Clayton Theory}

Clayton (1956) attempted to prove Butler's theory by suggesting that the most common missing teeth were vestigial organs and have no value for modern man in the evolutionary process. He examined 3557 subjects and noticed that the most posterior tooth of a tooth group (incisors, premolars and molars) was the most frequently missing in the sample . [50]Some scientists believe that future man's dentition will only have one incisor, one canine, one premolar and two molars for each quadran. This hypothesis needs evidence of a link of more favourable improvement in health with the reduction in teeth number.he first 2 . These lost teeth would have been farthest from the key tooth and in an evolutiona.[60] 


\section{Compensatory Tooth Size Interactions Model (1971)}

Sofaer et al., (1971) suggested that "if a tooth which developed early was larger than the average size, then its later developing adjacent tooth would have a higher chance to be smaller than normal, and vice versa" .[61] According to these authors, the reduction in tooth size and tooth agenesis is a compensatory interaction between tooth germs during development (e.g. lateral incisors develop after the centrals and their initiation depends on the availability of essential local needs. The absence or reduction in size of the teeth on one side induces a compensatory increase in size of the teeth of the contra-lateral side .[61]

\section{Multi Factorial Model Of Dental Anomalies Of Tooth Number And Size (1974)}

Brook, in his hypothesis, proposed a model where genetic factors play a major part in dental anomalies of tooth number and size, with an influence also from environmental factors .[61,62] This model includes two separate curves for males and females, the two tails of the curves demonstrate the relationship between different dental anomalies: tooth agenesis and microdontia lie at one end and supernumerary and macrodontia at the other end. Bailit (1975) suggested a polygenic model and agreed with Brook in his hypothesised model; both suggested that tooth agenesis illustrates the quasi continuous trait. $[55,62,63,64]$ According to both, the variable expression of tooth agenesis reports especially in monozygotic twins, supports the hypothesis of this model .[61-66] Fig.5

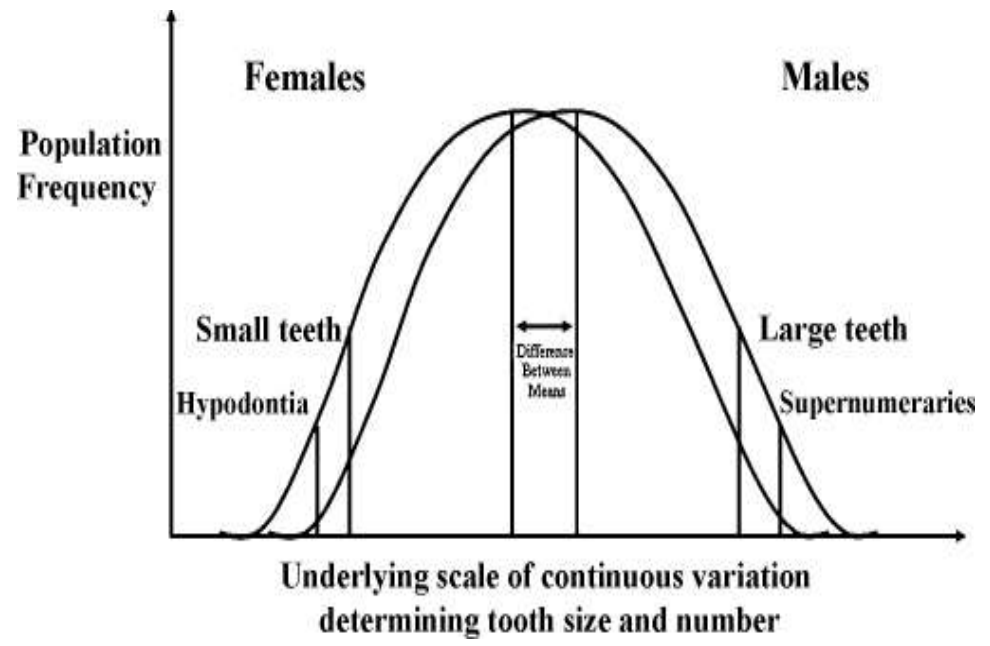

Fig.5 : Multifactorial model with superimposed thresholds that explains the relationship between tooth size and missing or extra teeth in males and females. The figure is based on one presented originally by Brook

\section{Anatomical model (1988)}

Svinhufvud et al have explained the selectivity of tooth agenesis in terms of an anatomic rather than an evolutionary model. These researchers suggested that certain regions during tooth development are more susceptible to epigenetic influences and hence agenesis. For example, the most frequently missing or variably sized tooth in the maxilla, the upper lateral incisor, develops in the area of the embryonic fusion between the lateral maxillary and medial nasal processes. In the mandible, permanent tooth agenesis occurs most frequently in the area of the second premolar. This corresponds to the distal end of the primary dental lamina, and because of its susceptibility to agenesis, this area is called a "fragile" site.[67] Interestingly, however, this site of mandibular agenesis appears specific for permanent dentition; the loss of second primary molars is rare.25A third site where tooth agenesis occurs frequently is the area where the 2 lower central incisors develop. Here, the fusion of the 2 mandibular processes is required to form the midline of the future mandible. This midline region is likely to be another fragile site.[67]

\section{Neural developmental fields model (1997)}

In this model Kjaer (1997) related the function of peripheral nerves to tooth agenesis. He proposed neural developmental fields in the maxilla and mandible as an explanation for the common sites of tooth agenesis. His hypothesis was based on the lack of innervation in the location of tooth agenesis. The area where the innervation ends in any of these fields (incisor field, canine/premolar, and molar field) is more likely to manifest tooth developmental failure .[68] 
The above models suggested different explanations for tooth agenesis phenomena. However, though they could apply to some mild phenotype, they failed to apply to the severe phenotype and in tooth agenesis phenotypes with variable penetrance in which one side was affected more than the other among the affected members. [68]

\section{Discussion}

Dental aplasia is a result of disturbances during the early stages of development and is suggested as a mild dysplastic expression of the ectoderm When a primary tooth is congenitally absent, its permanent counterpart might also be missing. Genetics plays a crucial role in congenital dental aplasia, as confirmed by studies on monozygotic twins.[1] Interestingly, the pattern of Congenitally missing teeth can differ between monozygotic twins, possibly pointing to additional underlying mechanisms, such as epigenetic factors which might be implied by simultaneous occurrence of two anomalies. [38,39] This multifactorial etiology can include environmental factors as well, since a combination of environmental and genetic factors might contribute to the occurrence of dental agenesis These include infection, trauma and drugs, as well as genes associated with about 120 syndromes, such as cleft lip, cleft palate or both, ectodermal dysplasia and Down, Rieger and Book syndromes A possible general explanation is that except in hereditary cases, Congenitally missing teeth has greater occurrence likelihood when the dental germ is developing after the surrounding tissues have closed the space needed for the tooth development.[37,64] Other investigations demonstrated that delays in tooth development and reductions in tooth size correlate with advanced Congenitally missing teeth. Both of these might accord with the terminal reduction theory. Furthermore, it is suggested that anterior agenesis may depend more on genes while posterior missing might be sporadic. [1,38,39,40]

The most supported etiological theory suggests a polygenic mode of inheritance, with epistatic genes and environmental factors exerting some influence on the phenotypic expression of the genes involved, which this can disturb the tooth germ during the initial stages of formation, i.e., the initiation and proliferation. The exact genetic mechanism is not known. Separate mechanisms might as well account for missing of each tooth .[1,41,42,43,44]]

Congenitally missing teeth can form in isolation as well. Isolated cases are more common than syndromic type and might be familiar or sporadic. The isolated condition can follow autosomal dominant, autosomal recessive or X-linked patterns of inheritance, with remarkable variation in both penetrance and expressivity.[1,37] Different subphenotypes of dental agenesis might be probably caused by various genes. Mutations in genes such as MSX, PAX9 or TGFA might cause Congenitally missing teeth in different racial groups. Among the homeobox genes, MSX1 and MSX2 play an important role in mediating direct epithelialmesenchymal interactions during craniofacial bone and tooth development. [1,37,38,39] The autosomaldominant Congenitally missing teeth might be correlated with a mutation in the MSX1 and PAX9 genes. MSX1 mutations affect predominantly the second premolars and third molars, sometimes in combination with other types of teeth like the first molars. On the other hand, in more common cases of incisor-premolar type of dental agenesis, MSX1 is less likely to play a role as the causative locus for this type of Congenitally missing teeth. In addition, PAX9 and TGFA are associated with congenital missing by interacting between MSX1 and PAX9. A recent study showed a novel mutation in MSX1 gene responsible for Congenitally missing teeth of the second premolars and third molars only. [1,37.41,42,43,44]

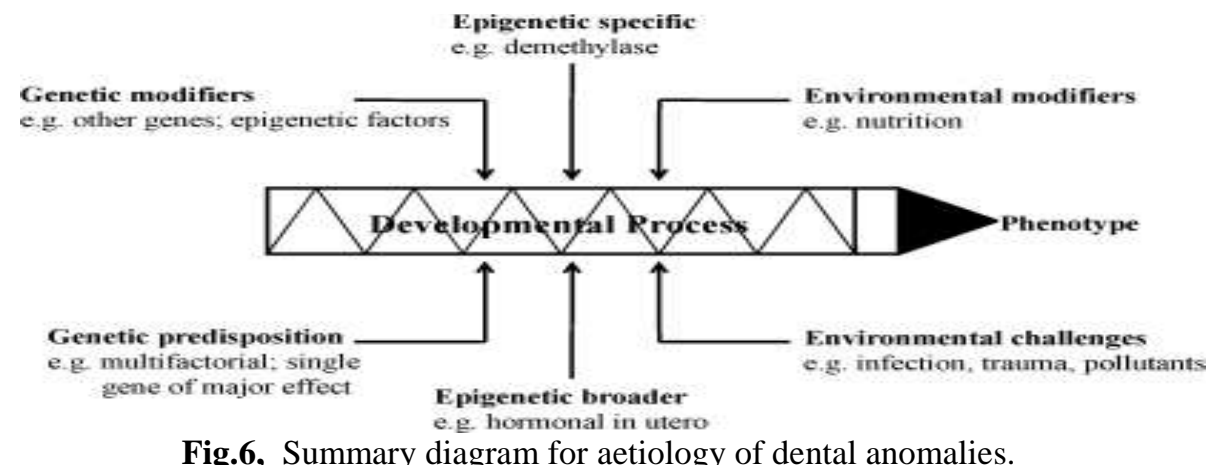

Anomalies of tooth number may not be isolated conditions, but may have wider associations in the development of the dentition, including tooth size. This study was aimed to examine the links between hypodontia, supernumerary teeth and crown size, considering the effect on the development of the whole dentition and to increase the understanding of the aetiology of these conditions. [69,70] Further, it has shown that the greater the number of the missing teeth, the smaller the tooth size.[70,71] The hypodontia patients also showed higher variability in tooth dimensions than that found in the control group.[71,72] Patients with supernumerary teeth had larger teeth than seen in the controls, with the greatest differences in the mesiodistal dimensions. In patients both hypodontia and supernumerary conditions, the differences in tooth size were 
generalized throughout the dentition. In anomalies of tooth number, the size of the teeth was also involved. In patients with hypodontia and supernumerary teeth conditions, the crown size of the whole dentition was affected. These findings are compatible with a multifactorial aetiology of these conditions.[71,72,73] Numerous epidemiological studies of hypodontia have shown the distributions of hypodontia in an adolescent sample of American blacks with a comparable sample of whites. The prevalence of people with missing teeth is significantly lower in blacks than in whites, as is the number of missing teeth per person. Significant sex differences were found only for the third molars (absence more common in females), and the sex differences were greater in whites than in blacks.[6,9

The congenital absence of teeth is a complex condition affecting several parameters of oral development.[39,40] This was the first study which was done to measure tooth crown dimensions by using image analysis in a family with hypodontia, in whom the mutation was identified, and to compare them with a control group. The majority of tooth types throughout the dentition were significantly smaller in the family members with hypodontia than in the control group, for all parameters which were measured.[69-75] The significantly smaller tooth crown dimensions which were recorded in the affected family members showed that the effect of the PAX9 mutation was seen not only in the congenitally missing teeth, but also in smaller crown sizes throughout the dentition[38-42].

The developmental absence of teeth (hypodontia) was reported to be associated with characteristic morphological changes in the teeth, alveolar volume deficiencies, and skeletal jaw malrelationships. The reports on the management of the patient with oligodontia by using adhesive techniques and new restorative materials, represent the current options in the management of the dental rehabilitation of young patients with oligodontia.[1] In familial human hypodontia the congenital absence of teeth is one of the commonest developmental abnormalities which are seen in human populations. Familial hypodontia or oligodontia represents an absence of the varying numbers of primary and/or secondary teeth as an isolated trait. While much progress has been made in understanding the developmental basis of tooth formation, the knowledge on the aetiological basis of the inherited tooth loss remains poor.[38-44] The study of mouse genetics has uncovered a large number of candidate genes for this condition, but mutations in only three have been identified in human pedigrees with familial hypodontia or oligodontia: MSX1, PAX9 AND AXIN2. [1]This suggests that these conditions may represent a more complex multifactorial trait, influenced by a combination of gene functions, environmental interactions and developmental timing. Completion of the human genome project has made available the DNA sequence of the collected human chromosomes, thus allowing the localization of all human genes and, ultimately, the determination of their functions.[39-44]

Dental anomalies are caused by complex interactions between genetic, epigenetic and environmental factors during the long process of dental development. This process is multifactorial, multilevel, multidimensional and progressive over time.[1] In this paper the evidence from animal models and from human studies is integrated to outline the current position and to construct and evaluate models, as a basis for future work. Dental development is multilevel entailing molecular and cellular interactions which have macroscopic outcomes. It is multidimensional, requiring developments in the three spatial dimensions and the fourth dimension of time. It is progressive, occurring over a long period, yet with critical stages. $[1,37,64]$ The series of interactions involving multiple genetic signalling pathways are also influenced by extracellular factors. Interactions, gradients and spatial field effects of multiple genes, epigenetic and environmental factors all influence the development of individual teeth, groups of teeth and the dentition as a whole. The macroscopic, clinically visible result in humans is a complex unit of four different tooth types formed in morphogenetic fields, in which teeth within each field form directionally and erupt at different times, reflecting the spatio-temporal control of development. Even when a specific mutation of a single gene or one major environmental insult has been identified in a patient with a dental anomaly, detailed investigation of the phenotype often reveals variation between affected individuals in the same family, between dentitions in the same individual and even between different teeth in the same dentition. [37]The same, or closely similar phenotypes, whether anomalies of tooth number or structure, may arise from different aetiologies: not only mutations in different genes but also environmental factors may result in similar phenotypes. Related to the action of a number of the developmental regulatory genes active in odontogenesis, in different tissues, mutations can result in syndromes of which dental anomalies are part.[1,37,64] Disruption of the antagonistic balance between developmental regulatory genes, acting as activators or inhibitors can result in dental anomalies. There are critical stages in the development of the individual tooth germs and, if progression fails, the germ will not develop further or undergoes apoptosis. The reiterative signalling patterns over time during the sequential process of initiation and morphogenesis are reflected in the clinical association of anomalies of number, size and form and the proposed models. An initial step in future studies is to combine the genetic investigations with accurate recording and measurement of the phenotype. They also need to collate findings at each level and exploit the accurate definition of both human and murine phenotypes now possible.[1,37,64] Fig.6 
Clinicians could be assisted by knowing the Congenitally missing teeth risk factors and its pattern of occurrence. As a general rule, if only a few teeth are missing, the absent tooth would be the most distal tooth of any given type. This applies to the maxillary laterals and the mandibular second premolars. On the other hand, it is suggested that the permanent maxillary first premolars, canines and first molars, which are likely to be more stable, have a relatively greater rate of Congenitally missing teeth in children with five or more teeth missing. [63,74,75]

\section{Conclusion}

Congenitally missing teeth is a prevalent multifactorial dental anomaly, usually appearing in females and in the permanent dentition. It is not conclusive whether it tends to occur more in the maxilla or mandible and also in the anterior versus posterior segments. It can accompany various dentoskeletal deformities, anomalies, or simply complications. Thus it should be attended by expert teams at the earliest possibility. The treatment not only improves speech and masticatory function, but also has psychological implications that may greatly help in regaining self-confidence. Specialist teams with access to traditional and evolving diagnostic and treatment technologies are best able to manage patients with complex treatment needs such as some of those presenting with hypodontia. The loss of teeth in young patients can cause aesthetic, functional, and psychological problems, particularly if the teeth of the anterior region are involved. Proper diagnosis can help in the management of these cases in a sportive way. Anyway, our patient did not have any problem which was related to speech, except the lisping of certain words, with which he was not bothered. The patient was motivated because of this and recalled for follow up.

\section{References}

[1]. Abu-Hussein M., Watted N., Yehia M., Proff P., Iraqi F. (2015) ; Clinical Genetic Basis Of Tooth Agenesis, Journal Of Dental And Medical Sciences ,14(12),68-77 Doi: 10.9790/0853-141236877

[2]. Holtta, P., Alaluusua, S., Saarinen-Pihkala, U. M., Peltola, J. \& Hovi, L. (2005a). Agenesis And Microdontia Of Permanent Teeth As Late Adverse Effects After Stem Cell Transplantation In Young Children. Cancer, 103, 181-90.

[3]. Nieminen, P. (2009). Genetic Basis Of Tooth Agenesis. J Exp Zool B Mol Dev Evol, 312b, 320-42

[4]. Vastardis, H. (2000). The Genetics Of Human Tooth Agenesis: New Discoveries For Understanding Dental Anomalies. Am J Orthod Dentofacial Orthop, 117, 650-6

[5]. Yonezu, T., Hayashi, Y., Sasaki, J. \& Machida, Y.( 1997). Prevalence Of Congenital Dental Anomalies Of The Deciduous Dentition In Japanese Children. Bull Tokyo Dent Coll, 38, 27-32.

[6]. Rolling, S. \& Poulsen, S. (2001). Oligodontia In Danish Schoolchildren. Acta Odontol Scand, 59, 111-2

[7]. Matalova, E., Fleischmannova, J., Sharpe, P. T. \& Tucker, A. S. (2008). Tooth Agenesis: From Molecular Genetics To Molecular Dentistry. J Dent Res, 87, 61723

[8]. Muhamad Abu-Hussein, Nezar Watted, Abdulgani Azzaldeen, Mohammad Yehia, Obaida Awadi, Yosef Abu-Hussein. (2015) Prevalence Of Missing Lateral Incisor Agenesis In An Orthodontic Arabs Population In Israel (Arab48). International Journal Of Public Health Research. Vol. 3, No. 3, 101-107

[9]. Muhamad Abu-Hussein, Nezar Watted, Ali Watted, Yosef Abu-Hussein, Mohammad Yehia, Obaida Awadi, Abdulgani Azzaldeen. (2015) Prevalence Of Tooth Agenesis In Orthodontic Patients At Arab Population In Israel. International Journal Of Public Health Research.Vol. 3, No. 3, 77-82

[10]. Sarnas, K. V. \& Rune, B. (1983). The Facial Profile In Advanced Hypodontia: A Mixed Longitudinal Study Of 141 Children. Eur J Orthod, 5, 133-43

[11]. Schalk-Van Der Weide, Y., Steen, W. H. \& Bosman, F.( 1992). Distribution Of Missing Teeth And Tooth Morphology In Patients With Oligodontia. Asdc J Dent Child, 59, 133-40

[12]. Goya, H. A., Tanaka, S., Maeda, T. \& Akimoto, Y. (2008). An Orthopantomographic Study Of Hypodontia In Permanent Teeth Of Japanese Pediatric Patients. J Oral Sci, 50, 143-50.

[13]. Ahmad W, Brancolini V, Ul Faiyaz Mf, Lam H, Ul Haque S, Haider M Et Al. (1998) A Locus For Autosomal Recessive Hypodontia With Associated Dental Anomalies Maps To Chromosome 16q12.1.Am J Hum Genet 62:987-991.

[14]. Aasheim B, Ögaard B. (1993) Hypodontia In 9-Year-Old Norwegians Related To Need Of Orthodontic Treatment. Scand J Dent Res 101:257- 260

[15]. Van Der Weide Ys, Prahl-Andersen B, Bosman F . (1993) Tooth Formation In Patients With Oligodontia. Angle Orthodontist M63:31-37

[16]. Brook Ah, Elcock C, Al-Sharood Mh, Mckeown Hf, Khalaf K, Smith Rn. (2002)Further Studies Of A Model For The Etiology Of Anomalies Of Tooth Numberand Size In Humans. Connective Tissue Research , 43:289-295.

[17]. Jorgenson Rj. Clinician's View Of Hypodontia. J Am Dent Assoc 1980;101:283-86

[18]. Grahnen H. (1956)Hypodontia In The Permanent Dentition. Aclinical And Genetical Investigation. Odontol Revy ; 7(Suppl.3): 1100 .

[19]. Muller Tp, Hill In, Petersen Ac, Blayney Jr. (1970) A Survey Of Congenitally Missing Permanent Teeth. J Am Dent Assoc 81: 1017.

[20]. Lapter M, Slaj M, Skrinjaric I, Muretic Z. (1998) Inheritance Of Hypodontia In Twins. Coll Antropol ; 22: $291-8$

[21]. Markovic M. (1982) Hypodontia In Twins. Swed Dent J Suppl ; 15: 153-62.

[22]. Tal H. (1981)Familial Hypodontia In The Permanent Dentition: A Case Report. J Dent ; 9: 260-4.

[23]. Pirinen S, Kentala A, Nieminen P, Varilo T, Thesleff I, (2001)Arte S. Recessively Inherited Lower Incisor Hypodontia. J Med Genet ; 38: 551-8

[24]. Chosack A, Eidelman E, Cohen T. (1975) Hypodontia: A Polygenic Trait, A Family Study Among Israeli Jews. J Dent Res ; 54: 16-9.

[25]. Mckeown Hf, Robinson Dl, Elcock C, Al-Sharood M, Brook Ah. (2002)Tooth Dimensions In Hypodontia Patients, Their Unaffected Relatives And A Control Group Measured By A New Image Analysis System. Eur J Orthod ; 24: 131-41. 
[26]. Cohen Mm. (1975)Congenital, Genetic, And Endocrinologic Influences On Dental Occlusion. Dent Clin North Am 19: 499-514.

[27]. Bixler D. (1997)Genetic Counseling In Dentistry. J. Dental Educ, ; 40:645-649.

[28]. Bailleul-Forestier I, Molla M, Verloes A, Berdal A. (2008) The Genetic Basis Of Inherited Anomalies Of The Teeth: Part 1: Clinical And Molecular Aspects Of Non-Syndromic Dental Disorders. Europ. J Med Genet, ; 51:273-291

[29]. Pemberton Tj, Das P, Patel Pi. (2004)Hypodontia: Genetics And Future Perspectives. Braz J Oral Sci ;4:695-706.

[30]. Agarwal A, Gundappa M, Miglani S, Nagar R. (2013) Asyndromic Hypodontia Associated With Tooth Morphology Alteration: A Rare Case Report. Journal Of Conservative Dentistry: Jcd. ;16(3):269-71.

[31]. Shinh As, Goyal D, Shaik Ja, Guram G. (2014)Familial Tendency Of Oligodontia In Three Generations: A Rare Case Report. Ijds ; 2(6):72-6.

[32]. Mostowska A, Biedziak B, Trzeciak Wh. (2006) A Novel Mutation In Pax9 Causes Familial Form Of Molar Oligodontia. Eur J Hum Genet ; 14: 173-9.

[33]. Lammi L, Halonen K, Pirinen S, Thesleff I, Arte S, Nieminen P. (2003)A Missense Mutation In Pax9 In A Family With Distinct Phenotype Of Oligodontia. Eur J Hum Genet. ;11(11):866-71.

[34]. Das P, Hai M, Elcock C Et Al. (2003) Novel Missense Mutations And A 288-Bp Exonic Insertion In Pax9 In Families With Autosomal Dominant Hypodontia.Am J Med Genet ;118a: 35-42.

[35]. Dhanrajani Pj(2002). Hypodontia: Etiology, Clinical Features, And Management. Quintessence Int ;33:294-302.

[36]. Stockton Dw, Das P, Goldenberg M, D'souza Rn, Patel Pi. (2000) Mutation Of Pax9 Is Associated With Oligodontia. Nat Genet ;24:18-9

[37]. Muhamad Ah, Azzaldeen A (2012) Genetic Of Non-Syndromic Cleft Lip And Palate. 1:510. Doi:10.4172/Scientificreports.510

[38]. Abdulgani A.,. Kontoes N., Chlorokostas G.,Abu-Hussein M (2015).;Interdisciplinary Management Of Maxillary Lateral Incisors Agenesis With Mini Implant Prostheses: A Case Report; Journal Of Dental And Medical Sciences ,14 (12) , 36-42 Doi: $10.9790 / 0853-141283642$

[39]. Abusalih A., Ismail H , Abdulgani A. , Chlorokostas G ., Abuhussein M .(2016); Interdisciplinary Management Of Congenitally Agenesis Maxillary Lateral Incisors: Orthodontic/Prosthodontic Perspectives, Journal Of Dental And Medical Sciences , 15 ( 1), 90-99 Doi: 10.9790/0853-15189099

[40]. Abu-Hussein M., Watted N., Abdulgani A., Borbélyb. (2015); Modern Treatment For Congenitally Missing Teeth : A Multidisciplinaryapproach; International Journal Of Maxillofacial Research, 1(2);179-190

[41]. Abu-Hussein M, Chlorokostas G, Watted N , Abdulgani A , Jabareen A, (2016),Pre-Prosthetic Orthodontic Implant For Management Of Congenitally Unerupted Lateral Incisors - A Case Report Journal Of Dental And Medical Sciences 2 .Vol 15 (2), 99-104 Doi: 10.9790/0853-152899104.

[42]. Abdulgani M. , Abdulgani Az ., Abu-Hussein M . (2016); Two Treatment Approaches For Missing Maxillary Lateral Incisors: A Case Journal Of Dental And Medical Sciences ,Volume 15, Issue 7 ,78-85 Doi: 10.9790/0853-150787885

[43]. Abu-Hussein M, Watted N, Abdulgani A (2015) Autogenous Tooth Transplantation - Reality Or Not. Int J Dent Health Sci 2: 722 730

[44]. Muhamad Abu-Hussein Et Al(2016), Congenitally Missing Lateral Incisors; Orthodontic, Restorative, And Implant Approaches. Int J Dent, 2:2, 71-84

[45]. Lin D, Huang Y, He F, Gu S, Zhang G, Chen Y, Zhang Y (2007). Expression Survey Of Genes Critical For Tooth Development In The Human Embryonic Tooth Germ. Dev Dyn. 236: 1307-1312

[46]. Wang Y, Groppe Jc, Wu J, Ogawa T, Mues G, D’souza Rn, Kapadia H (2009). Pathogenic Mechanisms Of Tooth Agenesis Linked To Paired Domain Mutations In Human Pax9. Hum Mol Genet. 15: 2863-2874.

[47]. Šerý O, Krejčí P, Bonczek O, Míšek I (2013). Modern Dna Methods In The Research Of Molecular Causes Of Hypodontia. Ortodoncie. 22: 173-178.

[48]. Peterkova R, Hovorakova M, Peterka M, Lesot H (2014). Threedimensional Analysis Of The Early Development Of The Dentition. Aust Dent J. 59 Suppl 1: 55-80.

[49]. Chinsembu Kc (2012). Teeth Are Bones: Signature Genes And Molecules That Underwrite Odontogenesis. J Med Genet Genomics. 4: $13-24$

[50]. Vastardis H, Seidman Jg, Seidman Ce (1998). A Human Msx1 Homeodomain Missense Mutation Causes Familial Tooth Agenesis. In: Davidovitch Z, Mah J, Editors. Biological Mechanisms Of Tooth Eruption, Resorption, And Replacement By Implants. Boston: Harvard Society For The Advancement Of Orthodontics; . 23-9

[51]. Holtta, P., Alaluusua, S., Saarinen-Pihkala, U. M., Peltola, J. \& Hovi, L. (2005a). Agenesis And Microdontia Of Permanent Teeth As Late Adverse Effects After Stem Cell Transplantation In Young Children. Cancer, 103, 181-90.

[52]. Holtta, P., Hovi, L., Saarinen-Pihkala, U. M., Peltola, J. \& Alaluusua, S. (2005b). Disturbed Root Development Of Permanent Teeth After Pediatric Stem Cell Transplantation. Dental Root Development After Sct. Cancer, 103, 1484-93.

[53]. Laluusua, S., Calderara, P., Gerthoux, P. M., Lukinmaa, P. L., Kovero, O., Needham, L., Patterson, D. G., Jr., Tuomisto, J. \& 54.Mocarelli, P. (2004). Developmental Dental Aberrations After The Dioxin Accident In Seveso. Environ Health Perspect, 112, $1313-8$.

[54]. Bailit, H. L.( 1975). Dental Variation Among Populations. An Anthropologic View. Dent Clin North Am, 19, 125-39.

[55]. Grahnen, H.(1956). Hypodontia In The Permanent Dentition. Odontol Revy, 7,1-100

[56]. Townsend, G., Rogers, J., Richards, L. \& Brown, T.\{ 1995). Agenesis Of Permanent Maxillary Lateral Incisors In South Australian Twins. Aust Dent J, 40, 18692

[57]. Simonson, M. A., Wills, A. G., Keller, M. C. \& Mcqueen, M. B.( 2011\}. Recent Methods For Polygenic Analysis Of Genome-Wide Data Implicate An Important Effect Of Common Variants On Cardiovascular Disease Risk. Bmc Med Genet, 12, 146.

[58]. Stahl, E. A., Wegmann, D., Trynka, G., Gutierrez-Achury, J., Do, R., Voight, B. F., Kraft, P., Chen, R., Kallberg, H. J., Kurreeman, F. A., Kathiresan, S., Wijmenga, C., Gregersen, P. K., Alfredsson, L., Siminovitch, K. A., Worthington, J., De Bakker, P. I., Raychaudhuri, S. \& Plenge, R. M. $\{2012\}$. Bayesian Inference Analyses Of The Polygenic Architecture Of Rheumatoid Arthritis. Nat Genet, 44, 483-9.

[59]. Clayton, J.M.( 1956). Congenital Anomalies Occuring In 3557 Children. J Dent Child, 23, 206-208.

[60]. Sofaer, J. A., Chung, C. S., Niswander, J. D. \& Runck, D. W. (1971). Developmental Interaction, Size And Agenesis Among Permanent Maxillary Incisors. Hum Biol, 43, 36-45

[61]. Brook, A. H. (1974). Dental Anomalies Of Number, Form And Size: Their Prevalence In British Schoolchildren. J Int Assoc Dent Child, 5, 37-53.

[62]. Brook, A. H. (1984). A Unifying Aetiological Explanation For Anomalies Of Human Tooth Number And Size. Arch Oral Biol, 29, 373-8. 
[63]. Muhamad Abu-Hussein , Nezar Watted, Viktória Hegedűs, Péter Borbély Abdulgani Azzaldeen (2015);Human Genetic Factors In Non-Syndromic Cleft Lip And Palate: An Update International Journal Of Maxillofacial Research 1,3,7-23

[64]. Brook, A. H., Griffin, R. C., Smith, R. N., Townsend, G. C., Kaur, G., Davis, G. R. \& Fearne, J.( 2009b). Tooth Size Patterns In Patients With Hypodontia And Supernumerary Teeth. Arch Oral Biol, 54 Suppl 1, S63-70.

[65]. Brook, A. H., Pitts, N. B. \& Renson, C. E. (1983). Determination Of Tooth Dimensions From Study Casts Using An Image Analysis System. J Int Assoc Dent Child, 14, 55-60.

[66]. Svinhufvud, E., Myllarniemi, S. \& Norio, R.( 1988). Dominant Inheritance Of Tooth Malpositions And Their Association To Hypodontia. Clin Genet, 34, 373-81.

[67]. Kjaer, I. (1997). Can The Location Of Tooth Agenesis And The Location Of Initial Bone Loss Seen In Juvenile Periodontitis Be Explained By Neural Developmental Fields In The Jaws? Acta Odontol Scand, 55, 70-2

[68]. Abu-Hussein M, Abdulgani A, Watted N, Zahalka M (2015) Congenitally Missing Lateral Incisor With Orthodontics, Bone Grafting And Single-Tooth Implant: A Case Report. J Dent Med Sci 14: 124-130.

[69]. Abdulgani A, Kontoes N, Chlorokostas G, Abu-Hussein M (2015) Interdisciplinary Management Of Maxillary Lateral Incisors Agenesis With Mini Implant Prostheses: A Case Report. J Dent Med Sci 14: 36-42.

[70]. Abusalih A, Ismail H, Abdulgani A, Chlorokostas G, Abu-Hussein M (2016) Interdisciplinary Management Of Congenitally Agenesis Maxillary Lateral Incisors: Orthodontic/Prosthodontic Perspectives. J Dent Med Sci 15: 90-99.

[71]. Abu-Hussein M, Chlorokostas G, Watted N, Abdulgani A, Jabareen A (2016) Preprosthetic Orthodontic Implant For Management Of Congenitally Unerupted Lateral Incisors - A Case Report. J Dent Med Sci 15: 99-104.

[72]. Abu-Hussein M, Watted N, Abdulgani A (2016) Managing Congenitally Missing Lateral Incisors With Single Tooth Implants. Dent Oral Craniofac Res 2: 318-324.

[73]. Muhamad Ah, Azzaldeen A (2012) Autotransplantation Of Tooth In Children With Mixed Dentition. Dentistry 2: 149.

[74]. Abu-Hussein M, Watted N, Abdulgani M, Abdulgani Az (2016) Tooth Autotransplantation; Clinical Concepts. J Dent Med Sci 15: 105-113.

\section{Correspondenceto:}

Abu-Hussein M uhamad, DDS, M ScD, M Sc, M Dent

Sci (Paed Dent), FICD,

123 Argus Street, 10441 A thens, Greece,

E-mail:abuhusseinmuhamad@gmail.com 